\title{
The teaching reform for the modular fixture experimental course by comprehensive measures
}

\author{
Pengzi Xu, Qiang Liu, Yong Hu, Qing Zou \\ The department of mechanical manufacturing and automation \\ The School of Mechanical Science and Engineering, \\ Jilin University \\ Changchun, China \\ e-mail:xupengzinew@qq.com
}

\begin{abstract}
The fundamental manufacture technology (FMT) is one of the most critical professional courses for the mechanical undergraduate students, and the modular fixture experimental course is an important part of this course. This paper presents the reform for this experimental course by making best of comprehensive measures, and the both of the practical and innovation ability of the students can be improved effectively by choosing this experiment course.
\end{abstract}

Key words-modular fixture, fundamental manufacturing technology, computer aided education, multimedia education, practical and innovation ability

\section{INTRODUCTION}

The fundamental manufacturing technology (FMT) is the vital component of the curricular system for the mechanical undergraduate students in the School of the Mechanical Science and Engineering of Jilin University, this course content is composed of the principle of the mental cutting, the processing method for different surfaces and machine tool, the machining accuracy and analysis, the mechanical process planning, and the design of the modular fixture. In order to improve the practical ability of the undergraduate students, besides the classroom teaching, the course also provides them with multiple practical teaching, such as experimental courses, curriculum project, and productive practice etc.

Modular fixture experimental course is one of its experimental courses. This paper aims to reform this experimental course by utilizing comprehensive measures, which include exhibiting the typical assembled modular fixtures, making use of computer technology and computer aided design (CAD) software to realize the virtual design and assemble of the fixtures, combining with other content of the FMT course, to inspire the study interest of the student and improve teaching quality.

\section{THE ORIGINAL EXPERIMENTAL CONTENT AND THE REFORM MEASURES}

\section{A. The original experimental content}

The original procedure of the modular fixture experimental course is as the follows:

(1).Each group of students read a drawing of a typical component, such as the shifting yoke of the gearbox, the gearbox cover, the transmission housing, and the crankshaft etc., all of which are the representative components of the automobile.

(2).The task of the students is to design a modular fixture for machining a flat surface, or a cylindrical surface or an inner bore surface of the above mentioned parts. So after reading the drawing of the part, the student should analyze that how many freedoms should be restricted for the surface that would be processed, and choose the relevant setting elements, gripping holder to limit the freedoms, or even choose the drill plate that is for the drilling a hole, if it is necessary.

(3).After selecting the necessary components of the fixture, assemble them in to a real modular fixture according to their analysis and design.

(4).The teacher appraises and rectifies the students work, then let them draw a sketch of the modular fixture they designed.

(5).Finish and hand in the report.

Although this experimental content is available, when it was initially set, there are also some shortcomings that cast negative impact on the teaching, such as:

(1).The students are not able to design the fixture effectively or the fixtures they designed are not feasible or even wrong because of less experience and inspiration, since the students have never seen the modular fixture before, and they are not even familiar with the typical parts of the modular fixture, not mention that they can design a fixture rapidly in the class.

(2).The course does not use the computer and software technology, so the students not only don't have interest in this course, but also they can't use those advanced manufacturing technology they have learnt in the practical application. But the computer aided education technology, the CAD software, and multimedia education are so helpful to the students to understand and master the modular fixture. Also, it is pretty easy to utilize the educational technology in this experimental course.

(3). Drawing the sketch of the fixture they designed is useless and a waste of time in the class, and it makes the students feel boring, because it is out of date, and this part can be replaced by the CAD software. Therefore, this content should be canceled. 


\section{B. The reform measures}

In order to arouse the student interest, improve the teaching quality and overcome the above drawbacks, the course content and procedure have been reformed as the follows:

\section{1) Adding heuristic education}

As the above mentioned, the students do not know well about the parts and the function of the modular fixtures, and have never seen the assembled ones before. Thus, in order to give the students enough inspiration, several display panels of fixtures and real assembled modular fixtures that used for machining the cylindrical surface, the flat surface, inner bore surface, and some typical parts have been bought, some videos about the modular fixture have also been made.

At beginning of the experimental class, these panels and real fixtures would be exhibited to the students, the teacher will discourse their components and function to them in detail by the material, and the students would a good visual knowing of the modular fixture, so it is very helpful to inspire the students to design a fixture.

2) . Adding virtual design and assemble technology

So as to arouse the interest of the students, this course makes best of the computer aided technology, and the classroom offer enough personal computers to the students. The data base of the workpieces and the modular fixture components have been installed by the CATIA software in these computers, the students can choose the suitable parts they need to design and assemble modular fixtures for the workpiece according to their design task, also, they can not only design one fixture, if they have any other plans, they can also design as many fixtures as they want by the software, if the time is permitted. Meanwhile, if the data base does not have the parts they need, the students can also design the parts by themselves, and then save them in the data base. The designed fixtures can be also saved in the data base, and the excellent ones could be the teaching material for the other students.

3) Combined the real assemble with virtual design

After the virtual design and assemble, the students should express their plans to the teacher, then the designed modular fixtures in the computer would be evaluated and corrected by the teacher, and the teacher can help the students choose the most feasible one to assemble by utilizing the real modular fixture parts. The course has bought enough sets of real modular fixtures, whose parts are corresponding to the ones in the data base. Then, the students can assemble the real parts into the modular fixture that they have designed by the computer.

4) Combined the designed work with the other content of the FMT course

The analysis and calculation of the position error is one of the most important content of the FMT course, but the students did not do well in this part, as the location mode of different workpieces are various, the method of error analysis is logical and very complex, also, all of the analysis and calculation process is done under the abstract condition, so it seems to be a little difficult for the students to understand it. Therefore, for the purpose to help the students to understand the analysis and calculation of the position error visually, it has been combined with the modular fixture experiment course.

Instead of drawing a sketch of the designed fixture, because of its useless and waste of time, the assembly diagram of the designed modular fixture can be output and printed by the computer and the typewriter separately, then the students should analysis the location mode of the designed fixture from the diagram, and measure all the relational dimensions of the workpiece and setting elements of the fixture. The dimension tolerance of the related dimension of the parts could be read from the drawings. So the position error of the modular fixtures that were designed by the students can be calculated according to the above information.

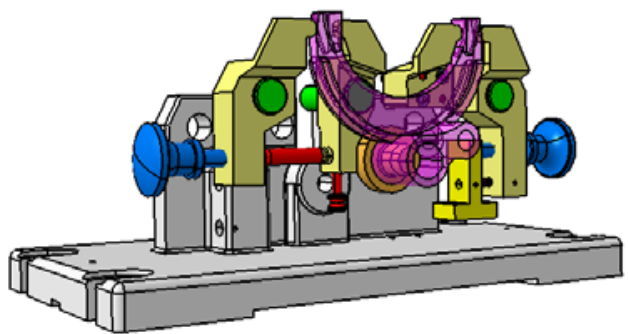

Figure 1. The typical virtual mode of the modular fixture designed by students

\section{CONCLUSION}

In summary, the modular fixture experimental course of FMT course has been reformed by comprehensive measures, which include adding the inspiration segment by exhibiting the panels, real fixtures, and videos, adding virtual design and assemble technology by computer aided education, combining the experiment content with the position error analysis and calculation, and printing the assemble diagram instead of drawing the sketch of the designed fixtures.

The reformed procedure of the modular fixture experimental course and its function are as the follows:

(1).The students visit and observe the display panels and the real assembled modular fixture, and the teacher explains the key point to them by the multimedia material. Consequently, the students get the well visual knowing of the modular fixture.

(2).The students analyze their task and design and assemble the as many modular fixtures as they can by the computer according to their analysis. This part can stimulate the interest of the students, and give them a chance to practice the CAD technology.

(3).The students talk about their plan to the teacher, and the teacher would evaluate and correct the students work, and help them to choose a most reasonable one to be assembled by the real fixture parts. It would be helpful to them to better understand the modular fixture. Figure 1 
shows one typical virtual model of modular fixture designed by the students.

(4).The students assemble the real modular fixture they designed by the real fixture parts, which can improve their practical ability.

(5).The students output and print the assemble diagram of the designed fixture by the computer and typewriter, and then analyze the location mode of the fixture, then measure the related dimension of the workpiece and the setting elements, then analyze and calculate the position error of the modular fixture they designed. This part combines another content of the FMT course, which is also one of the difficulties of the course, and the students are not good at it, so combining it with this experimental course can help students comprehend the method of the analysis and calculation of the position error.

(6).Finish and hand in the report.

\section{ACKNOWLEDGEMENT}

The authors must be grateful for the financial support from the Department of Science and Technology of Jilin Province (201201026).

\section{REFERENCE}

[1] Junyi Yu, Qing Zou, The fundamental manufacturing technology, Machinery Industry Press, 2009

[2] Jin Cai, Research on Integrated Modeling of Modular Fixture Components Base, Ph.D dissertation, Hebei University of Technology, 2009

[3] Gaoliang Peng, Xin-hua Liu,Weida Wang, Modular fixture assembly design system based on virtual reality, Computer Integrated Manufacturing Systems, vol.14 No.5 pp.984-990, 2008

[4] Xiangyun Zuo, Study for Computer Aided Design and Assembly Method of Modular Fixture, Master thesis, Taiyuan University of Science and Technology, 2010

[5] Yuguang Wu, Shuming Gao, Zichen Chen, Geometric theory on automated modular fixture planning, Chinese journal of mechanical engineering, vol.8 No.1 pp.117-122, 2002

[6] Zhigang Xu; Kezheng Huang; Xing Ai; Yuqi Liu, Reserch on the system of modular fixture design aotomation, Chinese journal of mechanical engineering, vol.36 No.12,2000

[7] Yaoxiang Zhu, Yiming Rong, Jian Zhu, Yong Bai Luo,Zhenbi, Study on computer aided modular fixture design system, Chinese journal of mechanical engineering, vol.5,1994

[8] Hongjun Chen, Detao Zheng, Jian Sun, Xin Chen, Hanwu He, Virtual design of modular fixture assembly, Machinery design and manufacture, No.6(12) pp39-41,2000

[9] Hongwei Liu, The Research and Development on Computer-aided Fixture Design system, Master thesis, North China Electric Power University,2007

[10] Yong Hu, Qing Zou, Pengzi Xu, Yonglan Tao, The teaching reform of manufacture technology basis course based on network, vol.30 No.4 pp105-107,2011 